\title{
Light blue crest sign visualized by magnifying endoscopy in normal colonic mucosa
}
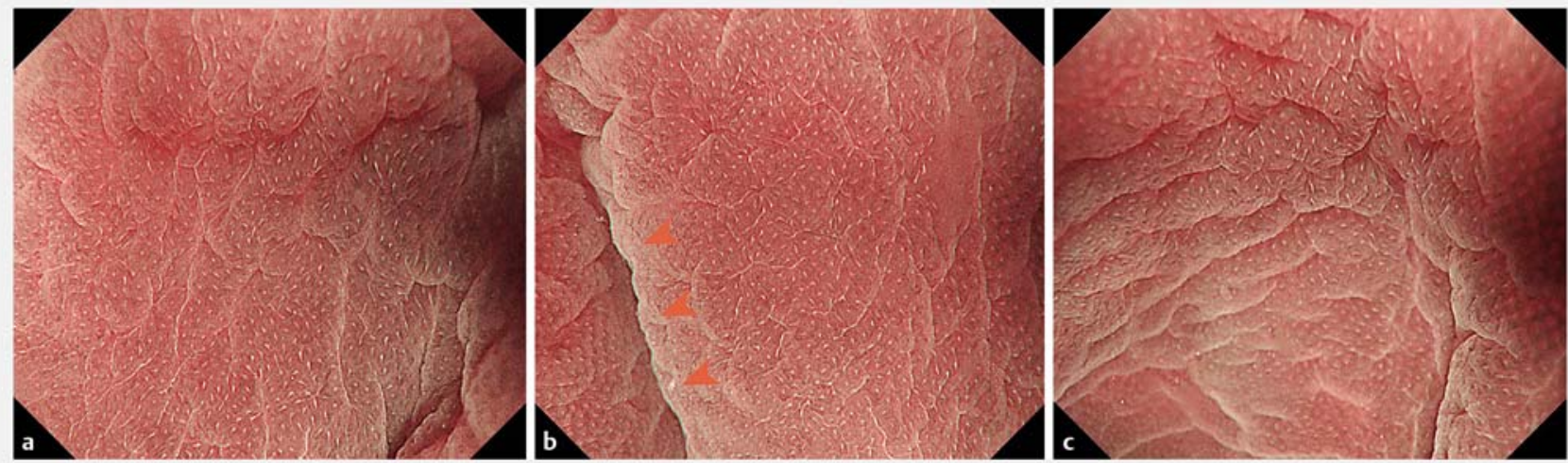

- Fig. 1 Normal colonic mucosa by magnifying endoscopy and narrow-band imaging. a The colon was partially collapsed and innominate grooves reappeared. Light blue crest (LBC) signs could be seen in the innominate grooves and crypt openings. b The long, linear, LBCs could be seen in tangential view of colonic mucosa (arrowheads). c Dot- or rod-like LBCs could be seen in partial crypt openings.

Light blue crest (LBC), defined as a fine, blue-white line on the crests of the epithelial surface/gyri, is always discerned at the edge of marginal crypt epithelium [1]. The optical sign has high specificity for the endoscopic identification of gastric intestinal metaplasia [1]. It can only be visualized by magnifying endoscopy with narrow-band imaging or blue-laser imaging, and is caused by the reflectance of short-wavelength light (400-430 nm) on the microvillous surface of the epithelium [1,2]. LBC is naturally seen in normal small intestine due to regular microvilli arrangement [1]. Herein, we describe the $\mathrm{LBC}$ sign in normal colonic mucosa. Colonic screening (GIF-H290Z; Olympus, Tokyo, Japan) was performed in a 40-year-old healthy man. When the colonic mucosa was fully dilated, the LBC sign could hardly be found. However, when the colon was partially collapsed and innominate grooves reappeared, creating a finely nodular surface, long linear LBCs could be seen in the corresponding grooves ( $>$ Fig. 1 a, $>$ Video 1 ) or in tangential view of the colonic mucosa ( Fig. 1 b). Dot- or rod-like LBCs could also be found in partial crypt openings ( Fig. $1 \mathrm{c}$ ).

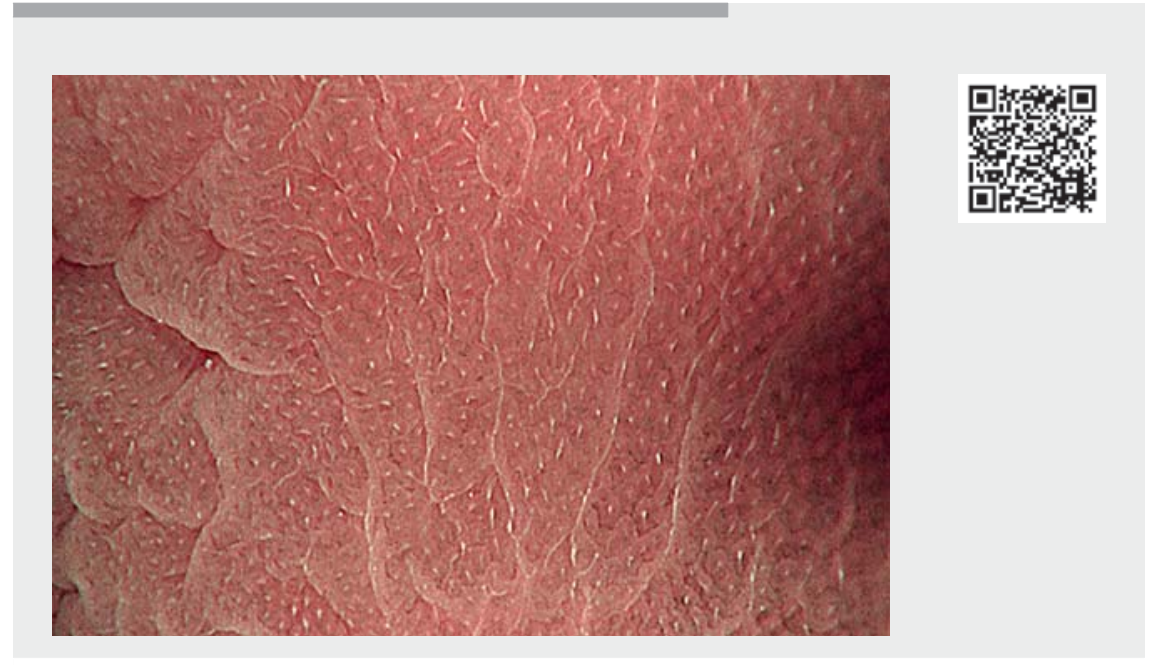

Video 1 Light blue crest (LBC) sign is naturally seen at the edge of the villi in normal duodenum. Innominate grooves reappear in the colon following partial collapse. Linear, dot- or rod-like LBCs can be seen clearly in innominate grooves and crypt openings.

Numerous crypts of Lieberkühn and innominate grooves were observed on the surface of colonic mucosa by scanning electron microscopy [3]. Examination by light microscopy of sections treated with hematoxylin and eosin and with immunohistochemical stains revealed that several crypts of Lieberkühn opened into innominate grooves (> Fig. 2) [3]. Inno- minate grooves and the upper part of Lieberkühn crypts were lined by mature absorptive cells [3], which possessed closely packed regular microvilli [4]. In tangential view of colonic mucosa (namely, perpendicular view of microvillous longitudinal axis), LBCs can be obtained by magnifying endoscopy, with regular microvilli illuminated by narrow-band 


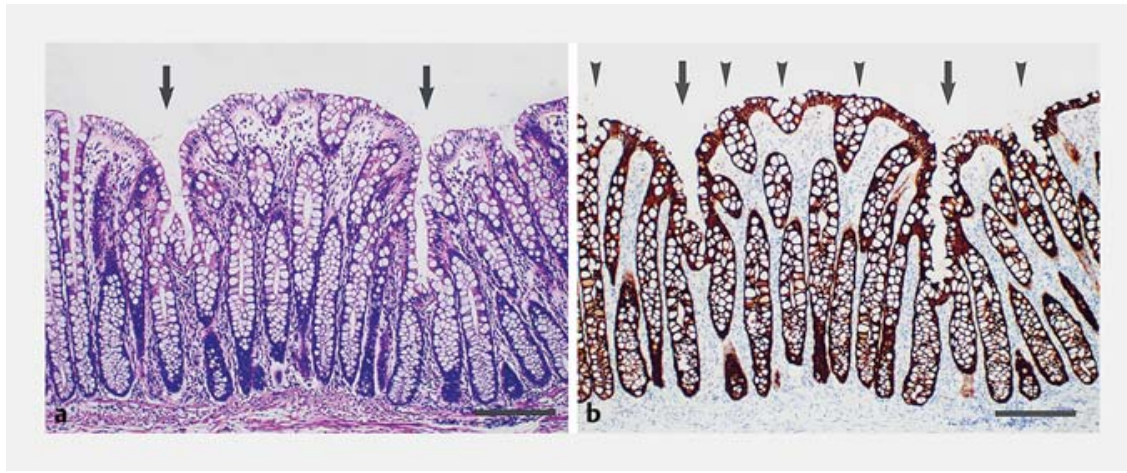

- Fig. 2 Histological examination. a Hematoxylin and eosin staining revealed several crypts of Lieberkühn opening into innominate grooves (arrow) (×100; bar $200 \mu \mathrm{m})$. b Villin immunohistochemical staining. Villin is a structural protein of microvilli. Positive areas represent the distribution of microvilli on the surface of colonic epithelial cells in innominate groves (arrow), crypts (arrowhead), and between crypts (×100; bar $200 \mu \mathrm{m})$.

blue light. No LBCs can be seen in normal gastric mucosa due to the lack of regular microvilli in foveolar epithelium [5].

Endoscopy_UCTN_Code_CCL_1AD_2AJ

\section{Competing interests}

The authors declare that they have no conflict of interest.

\section{The authors}

Cong Yuan ${ }^{*}$ ๑, Xue-Mei Lin²,3*, Hui-Li Zhu4, Yan $\mathrm{Ou}^{4}$, Qian Cheng ${ }^{4}$, Jun-Xiu Li ${ }^{4}$, Juan Liao ${ }^{4}$

1 Department of Gastroenterology, Affiliated Hospital of North Sichuan Medical College, Nanchong, Sichuan, China

2 Department of Pathology, Basic Medical College of North Sichuan Medical College, Nanchong, Sichuan, China

3 Department of Pathology, Affiliated Hospital of North Sichuan Medical College, Nanchong, Sichuan, China
4 Department of Gastroenterology, West China Forth Hospital, West China School of Public Health, Non-communicable Diseases Research Center, West China-PUMC C.C.

Chen Institute of Health, Sichuan University, Chengdu, China

\section{Corresponding author}

\section{Juan Liao, PhD}

Department of Gastroenterology, West China Forth Hospital, Sichuan University, No.18, Section 3, Renmin South Road, Chengdu 610041, China

juanliao@scu.edu.cn

\section{References}

[1] Uedo $\mathrm{N}$, Ishihara R, lishi $\mathrm{H}$ et al. A new method of diagnosing gastric intestinal metaplasia: narrow-band imaging with magnifying endoscopy. Endoscopy 2006; 38: 819-824

[2] Chen H, Liu Y, Lu Y et al. Ability of blue laser imaging with magnifying endoscopy for the diagnosis of gastric intestinal metaplasia. Lasers Med Sci 2018; 33: 1757-1762

[3] Riddell RH, Levin B. Ultrastructure of the "transitional" mucosa adjacent to large bowel carcinoma. Cancer 1977; 40: 25092522

[4] Helander HF, Fandriks L. Surface area of the digestive tract - revisited. Scand J Gastroenterol 2014; 49: 681-689

[5] Takagi T, Takebayashi S, Tokuyasu K et al. Scanning electron microscopy on the human gastric mucosa; fetal, normal and various pathological conditions. Acta Pathol Jpn 1974; 24: 233-247

\section{Bibliography}

Endoscopy 2022; 54: E498-E499

DOI 10.1055/a-1625-4859

ISSN 0013-726X

published online 8.10 .2021

(c) 2021. Thieme. All rights reserved.

Georg Thieme Verlag KG, Rüdigerstraße 14, 70469 Stuttgart, Germany

\section{ENDOSCOPY E-VIDEOS}

https://eref.thieme.de/e-videos

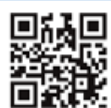

Endoscopy E-Videos is an open access online section, reporting on interesting cases and new techniques in gastroenterological endoscopy. All papers include a high quality video and all contributions are freely accessible online. Processing charges apply (currently EUR 375), discounts and wavers acc. to HINARI are available.

This section has its own submission website at https://mc.manuscriptcentral.com/e-videos

\footnotetext{
* These authors contributed equally to this work.
} 